CORRECTION

https://doi.org/10.1038/s41586-019-1225-0

\section{Author Correction: Metabolic reprogramming by the $S$-nitroso-CoA reductase system protects against kidney injury}

Hua-Lin Zhou, Rongli Zhang, Puneet Anand, Colin T. Stomberski, Zhaoxia Qian, Alfred Hausladen, Liwen Wang, Eugene P. Rhee, Samir M. Parikh, S. Ananth Karumanchi \& Jonathan S. Stamler

Correction to Nature https://doi.org/10.1038/s41586-018-0749-z, published online 28 November 2018.

In Fig. $1 j$ of this Letter, one data point was inadvertently omitted from the graph during reformatting for publication for the acute kidney injury (AKI), double knockout $(-/-)$, S-nitrosothiol (SNO) condition at a nitrosylation level of $25.9 \mathrm{pmol} \mathrm{mg}^{-1}$, and the legend stated that statistical significance was assessed using one-way analysis of variance (ANOVA) with Tukey's post hoc test, but in fact the value originally shown $(P=0.0221)$ was determined by Fisher's test. Analysis of these data using Tukey's test after instrument normalization for test-day (as per original experimental design; see the original Supplementary Information) yields a significance value of $P=0.0032$. The Supplementary Information for this Amendment contains the originally published Source Data for Fig. 1 j sorted by test-day. These changes do not affect the conclusions of this experiment. Figure 1 of the original Letter and its Source Data have been corrected online.

Supplementary information is available in the online version of this Amendment.
Original Fig. $1 \mathrm{j}$

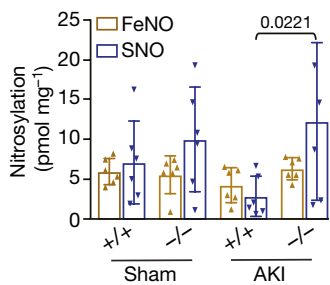

Corrected Fig. 1j

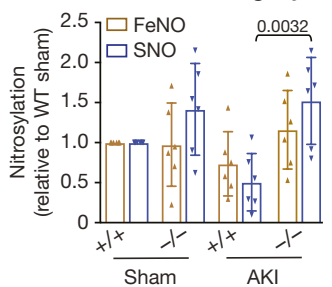

Fig. 1 This figure shows the original Fig. 1j (with missing data point, and analysed using Fisher's test) and the corrected Fig. $1 \mathrm{j}$ (with data point added and analysed using Tukey's test). WT, wild-type. The normalized ratio of 1 represents approximately $7 \mathrm{pmol} \mathrm{mg}^{-1}$ SNO. 